\title{
A REVIEW ON EXHAUST SYSTEM USING CYCLONE SEPARATOR
}

\author{
Dr. R. Suresh Kumar \\ Head of the Department \\ Department of Mechanical Engineering, \\ Sri Eshwar College of Engineering, Coimbatore 641202, India \\ Gowtham K V, Ajeeth R, Blessvin Bruso P, Dhanushkrishna T \\ Department of Mechanical Engineering, \\ Sri Eshwar College of Engineering, Coimbatore 641202, India
}

\begin{abstract}
Cyclone separator is one of the most important devices for collecting tiny dust particles. This is commonly called as pollution control equipment. It was most probably used by various kinds of industries to prevent the harmful minute dust particles to control the dust pollution in the surroundings. Cyclone separators could collect the micron-size dust particles effectively. The cyclone separator works on the principle of centripetal force, inertia force, gravitational force to separate the dust particles from the air and collect it in a separate collection box. The maximum efficiency of the cyclone separator can be attained by reducing its pressure drop. There are seven geometrical parameters considered while designing the cyclone separator namely inlet height, inlet width, outlet diameter, conetip diameter, total height, body height, and cyclone body diameter. The design and fabrication of the cyclone separator were slightly complicated because of its complex structure and to attain the maximum efficiency for the application.
\end{abstract}

Keywords - Cyclone separator, Dust particles, Collection Box, Pollution control Equipment, CFD approach.

\section{INTRODUCTION}

Industries play a major role in the development of the nation's economy by establishing new products to the global market. They also increase job opportunities for people. Establishing new products to the market and making money and increasing the country's GDP is not only being the duties of the industry. They also possess duties such as preventing the environment from pollutants and making some contribution to society.

Many industries such as the chemical industry, pharmaceutical and agricultural industry, cement and mining industry, oil refinery and stone industries exhibit very minute harmful dust particles to the environment. The dust particles emitting from the industries creating a fatal effect on human bodies, especially heart, lungs, and skin diseases. For avoiding and controlling these problems, many industries came with new ideas to collect all the dust particles. They came with lots of equipment to collect dust particles. Most of the industries came up with the idea of using cyclone separators to collect dust particles and to make the atmospheric air pure for inhaling. Cyclone separator is one of the best dusts collecting device. It can be suitable for various applications and even very tiny particles are get collected in it. This separation of dust particles by the use of cyclone separator is mainly based on the density of the materials. The performance of cyclone separator is mainly affected by its high-pressure drop. At the same time, the improper geometric features of the cyclone increase or decrease the pressure drop. The Cyclone separator design consists of several parameters such as pressure drop, inlet width, inlet height, total height, and outlet diameter. Based on its shape, the centripetal force is created inside the cyclone separator which makes to strike the dust particles on the inner surface of the cyclone separator and because of gravitational pull highdensity particles get settled down into the collection box and the pure air is circulated outside to the surroundings.

\section{LITERATURE SURVEY}

\section{A. CYCLONE SEPARATOR}

Jun-Hyung Lim, et al., (2020) [1] This assessment presented additional deltas on the cone portion of a digressive rough wind separator and investigated its effect on collection capability and weight drop. When disintegrated was injected through the additional bays and clean air was mixed into the main channel, the cutoff size of particles decreased, appeared differently concerning that when mixing airborne through the primary narrows. In any case, it was revealed that there was a perfect flow rate through the additional inlets to redesign the twister separator's presentation. In the meantime, implanting airborne through not simply the additional deltas yet moreover the main sound disconnected a higher flowrate of airborne while demonstrating a near level of variety efficiency and weight drop appeared differently with those of the reference brutal breeze separator. Therefore, acquainting additional bays with the cone zone of a conventional superfluous savage breeze separator was depended upon to process a higher flowrate of disintegrated than the 


\section{International Journal of Engineering Applied Sciences and Technology, 2020 Vol. 4, Issue 11, ISSN No. 2455-2143, Pages 312-328 \\ Published Online March 2020 in IJEAST (http://www.ijeast.com)}

separator with the relative introduction, right now cost sufficiency. This investigation added four extra deltas to the cone area of a generally utilized extraneous gulf violent wind separator (reference typhoon separator) to structure an AIoCS typhoon separator. At the point, when dust airborne was infused uniquely through the extra gulfs while keeping up the AIoCS twister separator's flow rate at $30 \mathrm{l} / \mathrm{min}$, its cut-off size diminished by 0.5 micrometres contrasted with that of the reference violent wind separator. At the point, when clean air was infused through the AIoCS violent wind separator's unique bay at $30 \mathrm{l} / \mathrm{min}$ while extra residue airborne was infused through the extra deltas, the AIoCS twister separator's cut-off size diminished by 0.5 micrometres or more contrasted and that of the reference tornado separator. Notwithstanding, when clean air was infused through the AIoCS typhoon separator's unique gulf, it's all outflow rate of airborne, which could be handled for various intentions, was lower than that of the reference cyclone separator. In the meantime, when dust airborne was infused through the AIoCS tornado separator's unique delta at $30 \mathrm{l} / \mathrm{min}$ while dust vaporized was all the while infused at $10 \mathrm{l} / \mathrm{min}$ or less through the extra deltas, the AIoCS typhoon separator indicated a comparative assortment productivity and weight drop contrasted with those of the reference violent wind separator and handled a higher flowrate of airborne. From these outcomes, introducing extra deltas to the cone area of a customary extraneous gulf twister separator, e.g., a typhoon separator utilized as a pre-separator for a PM10 or PM2.5 samplers, was relied upon to process a higher flow rate of vaporized while keeping up indistinguishable execution, in this manner making its activity more financially savvy.

Li Qiang, et al., (2019) [2] Recreations and exploratory strategies were utilized to assess the presentation of indented vortex discoverers in a cyclone separator with a distracting delta. The vortex discoverer was cut diagonally. Four sorts of violent wind separators with various vortex discoverer structures were considered. One of the separators didn't have a cut at the vortex discoverer, while different separators had cuts situated at $45^{\circ}, 135^{\circ}$, and $225^{\circ}$ inverse to the channel of the separator. A shaky Reynolds stress transport model (RSM) was executed for the gas-stage stream field, and a discrete stage model (DPM) was embraced for the molecule stage. Considering the hilter kilter stream field in the separator with a digressive gulf, the appropriation of hub speed, unrelated speed, and spiral speed along the circuit were talked about, and the impact of the indented vortex discoverer on the stream field in the separator has appeared in detail. The evaluation efficiencies of the four models were thought about. The reproduction results show that the partition exhibitions of the violent wind separators with indented vortex discoverers were significantly improved over the detachment execution of the first separator. At the point, when the cut position was found $225^{\circ}$ inverse to the channel, the partition execution of the violent wind separator improved most clearly. Examinations were done to affirm the upsides of scored vortex discoverers. The test results show that a separator with an indented vortex discoverer at $225^{\circ}$ can expel particles more prominent than 3.5 micrometres in width, while an ordinary separator can evacuate just particles more noteworthy than 8 micrometres in measurement. Besides, the complete detachment effectiveness of the first separator was 0.73 , while the absolute division proficiency of the improved separator was 0.76 .

Yanqin Mao, et al., (2019) [3] To improve the presentation of the division gadget, a novel sort of violent wind separator was structured. The impacts of the structure parameters and activity parameters on pressure drop and partition effectiveness are researched dependent on symmetrical trial through the test stage. Moreover, the interior stream attributes in the upgraded twirl generator are examined by numerical re-enactment. In like manner, the weight drop of the enhanced whirl generator was 170.9 $\mathrm{Pa}$ and its detachment proficiency was $68.23 \%$ when extraction proportion was $5 \%$. The cutting-edge shape and the edge outlet edge can impact the weight drop and productivity. The breadth of directing cones affects pressure drop, yet has no impact on the proficiency. At the point when the extraction proportion was expanded to $10 \%$, the productivity could be raised to $79.97 \%$, and it has no noteworthy increment at higher extraction proportion. The division proficiency can reach to $88.10 \%$ when it was utilized to isolate $180-250 \mathrm{um}$ sand. At last, the recreation uncovers that the stream highlights and dissemination in the enhanced whirl generator. In this way, the new type typhoon separator, which was relied upon to be applied in partition fields, has extraordinary genuine utilizing esteem. This work shows an investigation of a novel hub stream violent wind separator. Also, this separator fills in as a precleaner diminishing the end of the channel. The symmetrical trial was utilized to streamline the parameter. What's more, the impacts of the different twirl generators on the opposition and detachment execution are explored. The inward stream field of the upgraded whirl generator was re-enacted by FLUENT 18.0. Looking at other tornado separators, the weight drop was altogether brought down while the effectiveness was as yet keeping up an elevated level.

Mofarrah Masoumeh, et al., (2019) [4] The purpose of this examination was evaluating a scaled down scale gas twister execution with a body expansiveness of 10 $\mathrm{mm}$ to accumulate indoor fine particles. The structure of a rough wind requires constraining the weight drop and boosting the segment capability. All things considered and grade efficiencies, pressure drops, and cut sizes have been inquired about through a speculative model, multiplication, and exploratory examinations. The exploratory part was coordinated using an Electrical Low-Pressure Impactor (ELPI) device to check particle centre for stream paces of 10-13.3 (1/min). To look at the weight-drop and speed lead for different stream rates, COMSOL writing computer programs were utilized. The gained results from preliminary work have met the 


\section{International Journal of Engineering Applied Sciences and Technology, 2020 Vol. 4, Issue 11, ISSN No. 2455-2143, Pages 312-328 \\ Published Online March 2020 in IJEAST (http://www.ijeast.com)}

theoretical and generation results adequately. It has been avowed by all the got results that by growing the stream rate and thusly delta speed, the particle variety viability and weight drop increase while the cut size decreases. The procured by and large efficiencies for both hypothetical model and test work are good and the two outcomes show that by the expansion of stream rate, generally speaking, productivity increments too. Recreation and test results speak to, as the stream rate builds the weight drop additionally increments. No critical contrasts were seen between the re-enactment and test information.

Deng Guanle, et al., (2019) [5] Weight drop and collection profitability are used to survey the segment execution of the vicious breeze separator. Right now, they drove relative examination of twister models using response surface procedure (RSM), back causing neural framework (BPNN), and social event methodology for data dealing with (GMDH) frameworks to make perfect insightful rough wind models. In like manner, we drove multi-target improvement for expanding model and restricting model using genetic computation (GA). CFD was performed as opposed to the testing procedure to get the assessed characteristics for the showing of $\_p$ and $\eta$. The endorsement outcomes of CFD showed $0.5 \%$ and $2 \%$ botches for $\_p$ and $\eta$, exclusively, differentiated and the exploratory data. Second, the structure of preliminary (DOE) assessment for 10 tropical storm geometrical parameters was executed to secure the important geometrical information. Vortex pioneer separation across $D_{x}$, delta width $a$, channel height $b$ and cone stature $\mathrm{H}_{\mathrm{co}}$ essentially influence $\eta$ and p. In any case, affiliation impacts between the geometrical parameters have little effects. The fierce breeze models by RSM, BPNN, and GMDH subject to $25 \mathrm{CFD}$ getting ready to set were made. The farsighted introduction results by the twister models were taken a gander at by 25 CFD test set. The GMDH method achieved the best conjecture for_p $(\mathrm{R} 2=99.7, \mathrm{RMSE}=0.102) \mathrm{R} 2$ adjusted $=98.99, \mathrm{RMSE}$ $=0.0119$ ) than the RSM, BPNN tornado models. Besides, helplessness examination was performed to check the quantitative execution of tropical storm models. The results show that the weakness width of GMDH models achieved the best conjecture $(\eta: \pm 0.0065$, _p: \pm 0.0188 ). Finally, GA was applied to streamline the GMDH designs simultaneously. GA delivered 70 nonprevalent game plans. The reproducibility of five perfect centres was endorsed by using CFD. The trade-off perfect point showed improvement by $24.31 \%, 18 \%$ and $8.79 \%$ for pressure drop 50 and efficiency, exclusively.

Generally, utilization of violent wind separator, particularly in ventures, makes it not just one of the most intriguing subjects of papers these days [6-12], yet innovative work of it ought to follow essentially. The bizarre plan of this cyclone makes it increasingly helpful and utilitarian. This structure causes to frame a solid vortex stream in the typhoon, which builds the diffusive power following up on every molecule independently, expands the assortment effectiveness as well as decline the cut-off width. Along these lines, the reproduction of the fierce 3D stream has been done with the limited volume technique also, RSM choppiness model. The significant commitment of the complete violent wind pressure drop has happened in the upper area of tornado and guide channels, which increment the entered speed to the fundamental body of the twister. The outcomes speak to a uniform distribution of static weight designs at violent wind statures for every speed as well as a uniform flow field design in the typhoon. In comparison with normal twisters, due to the unique structure of gulf control channels, not just the passage speed of stream to the fundamental violent wind body has been expanded, yet the improper moving of stream after the passageway to the principle body toward the typhoon's top area doesn't happen. The cut-off measurement decreased by 0.2 micrometres in examination with the typical typhoon separator. With $20 \%$ expanding in the delta speed, the most extreme static weight in all segments increments by around $41 \%$, while the $20 \%$ decrease in the channel speed, lessens the greatest static weight in all areas by $55 \%$ to $58 \%$. A $20 \%$ expansion in speed contrasted with the underlying speed of $8.809 \mathrm{~m} / \mathrm{s}$, decreasing the slice off distance across to around 0.6 micrometres. A $20 \%$ lessening in delta speed, the partition proficiency was diminished by and large and expands the slice off distance across to 0.9 micrometres. The Stokes number littler than 1, ought to be communicated as a proper rule to choose between two primary parts of the EulerianLagrangian and Eulerian-Eulerian approach. In this paper, we examined a violent wind separator with manage channels and consequences for pressure drop and partition proficiency, numerically. Recreation of the violent stream with computational liquid elements (CFD) strategies has been finished utilizing the Finite Volume Method (FVM) and RSM disturbance model.

Wei Zhang, et al., (2019) [13] Exact desire for the tangled nonlinear relationship among the assessment adequacy, geometrical estimations, and working parameters reliant on limited test data was the best technique to design a high-capability tropical storm separator. Right now, the PCA-PSO-SVR model was proposed to predict the assessment viability of rough wind separators with the working parameters subject to 217 courses of action of preliminary data given in the composition. The exploratory data are pre-processed using the sporadic testing framework together with the institutionalization procedure and head section examination (PCA) from the start; thusly, the atom swarm improvement (PSO) computation was combined to propel the parameters for the assistance vector backslide (SVR), including the discipline factor C, parcel work parameter $\mathrm{g}$, and coldblooded disaster $\varepsilon$. Finally, the SVR model with the streamlined parameters was set up with $80 \%$ pre-treatment data, and the theoretical limit of the model was attempted with the remaining $20 \%$ data. The mean squared goof of the test sets was $6.948 \times 10-4$ with a relationship coefficient of 0.982 . The assessment results show that the PCA-PSO-SV designs had higher 


\section{International Journal of Engineering Applied Sciences and Technology, 2020 Vol. 4, Issue 11, ISSN No. 2455-2143, Pages 312-328 \\ Published Online March 2020 in IJEAST (http://www.ijeast.com)}

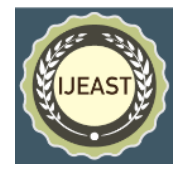

precision, better hypothesis limit, and more grounded force than the present models for anticipating the violent wind separator effectiveness for the situation with just a couple of test information. The PCA-PSO-SVR displaying strategy, which joins the primary segment examination, molecule swarm enhancement, and bolsters vector relapse calculation, was proposed to demonstrate the tornado proficiency utilizing the test information. The re-enactment results show that PCA, as a solo dimensionality decrease calculation, can adequately lessen the dimensionality of highlight space, wipe out incomplete commotion information, diminish the multifaceted nature of the model, and improve the speculation capacity of the model.

Nikolay Mikheev, et al., (2018) [14] This article contains data related to the investigation article entitled "Vicious breeze separator for a gas-liquid mix with high change thickness" This work was centred around the improvement of a convincing gas-liquid twister separator at a high movement thickness. Within the execution of the undertaking, the goal was to pick the schematic advancement of the separator gives the diminished special head of stream during the zones with extended liquid stage obsession to prevent the entrainment of secluded liquid and layer adversities. The paper shows the numerical performing data and test data allowed estimation of division capability of the separator.

Ilya Saushin, et al., (2018) [15] The assessment was centred around the progress of efficiency of a gas-liquid separator working at the heaviness of 2.5 Mega Pascal and high change thickness in the tornado body. The submitted schematic advancement of the separator gives the lessened novel head of gas during the zones with extended liquid stage centre and hinders the entrainment of disengaged liquid to the outlet authority. The gatherer containing a decreased joining channel and a remotely finned round pipe was arranged in a barrel moulded twister body. A cutting edge and-chamber stream conditioner were mounted upstream of the consolidating channel inlet. Distinct stream structure in the separator has been gotten from numerical examination reliant on Reynolds-found the centre estimation of Navier-Stokes conditions with anisotropic Reynolds stress unsettling influence model. The results were related to the estimation of the central execution traits of the separator and probability of dot entrainment and upwards gushing film. Test research on a 1:2 separator model allowed estimation of parcel capability and stream impediment coefficient of the separator. The effect was practiced due to especially convincing damping of the rapid head in the outlet channel of the separator. It was set up from numerical re-enactment of stream in the separator that there are no conditions for drop entrainment from the liquid film surface at the best stream pace of the mix, and the nearby divider dynamic head of upward streams doesn't outperform $30 \mathrm{~Pa}$. Such estimations of the dynamic head prevent conditions that trigger upward film streams from the lower base.
Seung-Yoon Noh, et al., (2018) [16] The point of this investigation was to build up another sort of violent wind separator that can expand assortment productivity while keeping up a low weight drop, and think about its assortment proficiency and weight drop with that of the general types of distracting tornado separators. The proposed separator joins a few little violent winds to one unrelated typhoon and was named the different auxiliaries prepared focal (MSEC) twister. Numerical examination and test outcomes indicated that the MSEC violent wind could expand assortment proficiency while all the while bringing down the weight drop. The outcomes show that backup violent winds altogether add to improving the presentation of the MSEC tornado. This investigation applied a parchment type plan to the most generally utilized distracting bay tornado and proposed another kind of twister separator, named as the MSEC typhoon, by appending a few backup tornados to the outside of the violent wind body. Two standard violent winds of various sizes were considered to look at twister execution. The aftereffects of the backup violent winds building up a steady precise interim between auxiliary violent winds and a consistent profundity of backup typhoons added in the focal tornado body demonstrated that the airborne stream rate in every auxiliary twister was the equivalent. The MSEC twister has potential for wide application to a few fields that require size arranging with a lot of mist concentrates.

S. Venkatesh, et al., (2018) [17] The tornado separator execution had been impacted by its highpressure drop. The essential geometric extents, for instance, outlet estimation, channel width, and delta stature and hard and fast height have been needed to decrease the weight drop and improve the presentation of tropical storm separator. These standard geometric characteristics had been changed with the guide of the plan of a preliminary system by the Taguchi strategy for decreasing the weight drop. This changed new arrangement produce low-pressure drop differentiated and the standard tornado separator. Furthermore, the combination profitability of the new structure was high when differentiated and standard hurricane separator. The weight drop, Euler number, cut-off estimation and capability of the standard and new twister separator had been differentiated and the delayed consequences of numerical and computational fluid components method (CFD). The Reynolds stress aggravation model and discrete stage model have been used for performing the rough wind separator in CFD. A commendable understanding had been gotten between these results. The most noteworthy geometrical parameters of the typhoon separator were channel stature and bay width, outlet distance across and complete tallness, which was the most part connected with the tornado separator's presentation. These parameters of the standard Shepherd and Lapple violent wind separator had been changed by the Taguchi technique. This changed new plan gives a low weight drop when contrast with the standard twister 


\section{International Journal of Engineering Applied Sciences and Technology, 2020 Vol. 4, Issue 11, ISSN No. 2455-2143, Pages 312-328 \\ Published Online March 2020 in IJEAST (http://www.ijeast.com)}

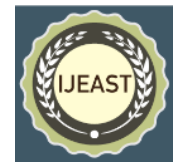

separator. Likewise, the assortment effectiveness of the new plan violent wind separator was high. So, the presentation of the violent wind separator had been upgraded.

Haolong Li, et al., (2018) [18] Hydrodynamic characteristics in a hurricane separator were reproduced by techniques for DEM-CFD. Reynolds stress unsettling influence model (RSM) was used to get gas unevenness. By changing the inlet point, the spreads of weight drop, digressive and centre speed of gas arrange were gained inside the tornado. Recreated results exhibit that the stream configuration involves two regions: hardship free vortex region and obliged the vortex zone. The negative inlet edge accomplishes a greater weight drop standing out from a positive channel edge. The segment adequacy and bearing of particles from re-enactment are gotten. The effects of straight edge and atom size on separation capability were estimated. The division adequacy was extended with the development of particle size, while the separation capability directly off the bat increases and subsequently declined as bay point changes from negative to positive. A comprehension between the numerical amusement and preliminary outcomes had been practiced in a twister separator. In this paper, the point by point stream data and execution of five diverse violent wind separators with various gulf edges are gotten with CFD-DEM. Reynolds stress model has been utilized to mimic the gas violent stream. Their legitimacy was affirmed by looking at the anticipated and estimated pressure drop and gas speed.

To sum up the portrayal of molecule size cut execution for streamlined violent winds, a piece of information-driven displaying approach utilizing two differed the corresponding system $\Psi 0.5=\mathrm{Cc} 1 / 2 \mathrm{dp} 0.5 / \mathrm{D}$ and Stk0.5 $=($ Ccepdp0.52vi $) /(18 \mu \mathrm{D})$ was proposed [1924]. This methodology related the worldwide impacting parameters including violent wind measurements, working conditions, and multiphase properties which have not ever totally included previously. The proposed models incorporated the outside geometrical measurements (tornado channel territory (abdominal muscle/D) and vortex discoverer distance across De/D) into an annular Reynolds number Rea $=\rho g Q(D-$ $\mathrm{De}) /[\mu(\mathrm{ab})]$, and utilized a unique body dimensionless parameter DB/D got from a proportionate volume strategy to portray impact of the typhoon body measurements: vortex discoverer length $\mathrm{S} / \mathrm{D}$, violent wind tallness $\mathrm{H} / \mathrm{D}$, chamber stature $\mathrm{h} / \mathrm{D}$ and molecule outlet width B/D. Results indicated that the proposed $\Psi 0.5$-based connection improves the prescient capacity and speculation execution contrasted with the other relating hypothetical and relapse models. Besides, the impact of twister measurements and working parameters on molecule cut off size was quantitatively tended to depend on the proposed model. The outcome may give a reference to execution appraisal, plan improvement and worldwide streamlining of streamlined tornados applied both the mechanical procedure and airborne testing. An improved and summed up demonstrating approach for twister molecule cut off size was created utilizing $\Psi 0.5-$ based and Stk0.5-based techniques, by exhaustive corresponding the worldwide violent wind measurements, working parameters and multiphase properties. The two dimensionless gatherings were utilized to portray the molecule cut off size dp0.5: annular gas Reynolds number Rea coupling with the outside geometrical parameters (stomach muscle/D2) and (1-De/D), and the body geometrical parameters DB/D dependent on successful volume technique, which indispensably mirrors the impacts of the twister measurements $\mathrm{S} / \mathrm{D}, \mathrm{H} / \mathrm{D}, \mathrm{h} / \mathrm{D}$ and $\mathrm{B} / \mathrm{D}$.

Kunakorn Poochinda, et al., (2017) [25] Sustainable power source was vitality that was gathered from inexhaustible assets, for example, daylight, wind, downpour, tides, waves, geothermal warmth and biomass. Biomass was elective fuel that can be combusted to get power in different enterprises. Inorganic compound ordinarily found in debris was related to the item gas after the burning. The gadget that was broadly used to isolate the debris out of item gas was violent wind because of the basic development, low support cost and high dependability which utilizing outward power. Since regular violent wind separators can't separate strong particles with an elite, the partition of particles in various novel tornado shapes has been researched in this investigation employing three measurements of computational liquid elements (CFD). The computational cross-sections with high goals of roughly 170,000-190,000 cells and (RNG) k- $\varepsilon$ model are utilized for stream investigation of the tornados. The reenactment results have been precisely contrasted and the test results from Azadi et al. At that point, the parameters in thought are the measurement of the gas outlet (vortex discoverer) and the edge of the gulf with the bay speed of $15 \mathrm{~m} / \mathrm{s}$ and strong volume part of $6 \times 105$. In this investigation, the precise CFD model for the reproduction of three-dimensional violent wind was created. The computational cross-sections with high goals of 170,000-190,000 cells and (RNG) k- $\varepsilon$ model were utilized for stream investigation. At that point, the impacts of typhoon vortex discoverer and bay edge on strong division were researched. The four new typhoon plans were thought about the outcomes (assortment proficiency, pressure drop, and stream conduct) with a standard twister, Stairmand tornado, from Azadi et al.

Adi Surjosatyo, et al., (2017) [26] Maker gas created from biomass gasification despite everything contains particulate debris. It was cleaned by utilizing a violent wind separator. Violent wind separator ability to 'catch' debris was described by its presentation known as assortment proficiency. One main consideration impacting violent wind separator execution is vortex discoverer measurement. A twister separator model with 2D standard design with $150 \mathrm{~mm}$ distance across has been made. The standard arrangement model issued to limit geometrical elements influencing typhoon separator 
execution other than vortex discoverer itself. Three varieties of length $(3 / 8 \mathrm{D} ; 5 / 8 \mathrm{D} ; 1 \mathrm{D})$ and three varieties of breadth $(35 ; 70 ; 85 \mathrm{~mm})$ of vortex discoverer are utilized to recognize their impacts on tornado execution. Test study and CFD recreation utilizing ANSYS Fluent were performed. Test study plans to discover vortex discoverer measurement consequences for assortment proficiency pattern, while CFD reproduction objective was to decide the speed profile of air inside twister separator. RNG $\mathrm{k}-\varepsilon$ for whirl ruled stream was picked as the choppiness model. Speed size inside violent wind separator was utilized to anticipate the assortment productivity pattern. Assortment effectiveness from the experiment was dictated by gauging strategy, for example, level of absolute mass of debris caught partitioned by all-out mass of debris entering tornado separator. Exploratory outcomes show that expanding vortex discoverer length and diminishing vortex discoverer distance across can build the assortment effectiveness. By and large, expanding vortex discoverer length and diminishing vortex discoverer breadth can likewise build the speed greatness of air inside tornado separator. Most elevated assortment proficiency is accomplished by utilizing vortex discoverer with $35 \mathrm{~mm}$ (1/4 D) in distance across and $1 \mathrm{D}(150 \mathrm{~mm})$ long. Given the exploratory outcomes, vortex discoverer with measurement of $70 \mathrm{~mm}$ has the most reduced assortment proficiency. Breadth $85 \mathrm{~mm}$ has a steady assortment of productivity on both the state of the wind stream rate. Measurement $35 \mathrm{~mm}$ with a length of $1 \mathrm{D}$ has the most noteworthy assortment productivity among different components of the vortex discoverer and it applies in both wind stream rate. In the wind current rate of 2.4 $\mathrm{m}^{3} / \mathrm{h}$, measurement $35 \mathrm{~mm}$ has a huge increment in assortment effectiveness by stretching of the vortex discoverer.

An-Ni Huang, et al., (2017) [27] A laminarizer including a bank of 15 chambers was amassed at the section of a twister. Tests and numerical re-enactments were performed to take a gander at the effect of the laminarizer on the weight drop over the twister, fragmentary parcel efficiency, gas irrelevant speed scattering, particle assignment at the way and half-cut size. Using the laminarizer insignificantly extended the weight drop over the tornado and to some degree diminished the gas diverting rate. The laminarizer balanced the air mass stream rate and atom stream scattering at the section. It extended the air mass stream rate through the outside bit of the way. At a channel gas speed of $11 \mathrm{~m} / \mathrm{s}$, presenting the laminarizer diminished the number of particles coursing through the internal bit of the entry by $20.2 \%$. Higher atom centres were found at the outside bit of the way, where the particles were even more viably trapped in the wake of entering the essential body. At a narrow gas speed of $11 \mathrm{~m} / \mathrm{s}$, using the laminarizer extended the weight drop over the tornado by $8.1 \%$ and diminished the half-cut size of the brutal breeze from 2.04 micrometres to 1.89 micrometres. The exhibition of a typhoon was assessed by tests and CFD reproductions when a laminarizer was introduced at its passage. The laminarizer somewhat expanded the weight drop over the violent wind as a result of the expanded gas-divider contact zone. A 4.0\%-8.1\% expansion in the weight drop over the typhoon was seen in the present framework. The laminarizer additionally diminished the extraneous gas speed. The most extreme digressive gas speed underneath the vortex discoverer was $2.1 \%-2.7 \%$ higher in the traditional violent wind than in the typhoon with the laminarizer

Dzmitry Misiulia, et al., (2017) [28] Four rule geometrical parameters of a deswirler (focus estimation, number of vanes, the stature of vanes and driving edge plot) for vicious breeze separators had been progressed using CFD and phony neural framework. The results showed that the most immense geometrical parameters of the deswirler were the number of vanes, the vane point, and the vane height. Another improved deswirler geometry was gotten using the genetic estimations and its ramifications for the stream field, pressure adversities, and twister collection adequacy was numerically looked into. The deswirler earnestly impacts the stream field inside a tornado. It essentially decreases digressive speeds in the vortex pioneer and only insignificantly (by $4.5 \%$ ) reduces most outrageous diverting velocities in the segmented zone. The deswirler moreover lessens the length of the inward vortex, redistributes reliably centre rates at the vortex pioneer outlet and prevents backward stream. A surrogate-based enhancement had been applied to get the ideal incentive as far as least absolute weight drop and weight misfortunes in the vortex discoverer for four principle deswirler geometric parameters went before by structure of examinations. At that point, numerical reproductions were performed trailed by fitting the surrogate models.

Hamed Safikhani, et al., (2017) [29] Numerical examination of the fluid stream and atom components was shown by the numerical methodology to depict the introduction of new arrangement tornado separators with one, two and three inconsequential bayous. The arrangement of this twister relies upon improving tropical storm execution by growing the vortex length. This vicious breeze differs from a customary storm with the division space. Instead of channel moulded part, the parcel space of this savage breeze contains an outer chamber and a vortex limiter. The Reynolds landed at the midpoint of Navier-Stokes conditions with Reynolds stress unsettling influence model (RSM) are settled by the usage of the restricted volume strategy reliant on the SIMPLE weight review figuring in the computational space. The Eulerian-Lagrangian computational procedure was used to predict particles following in the fierce breezes. The speed changes are reproduced using the Discrete Random Walk (DRW). In the results, the effects of the number of inlets on the differing huge parameters, for instance, pressure drop, collection adequacy, centre speed, and unevenness are analyzed and significantly discussed. The Reynolds found the centre estimation of 
Navier-Stokes conditions with Reynolds stress roughness model (RSM) were clarified by use of the constrained volume methodology reliant on the SIMPLE weight modification count in the computational space. The Eulerian-Lagrangian computational approach was used to foresee particles following in the tornados. The speed instabilities were reproduced using the Discrete Random Walk (DRW). In the results, the effects of the number of bayous on the unmistakable noteworthy parameters, for instance, pressure drop, assortment effectiveness, hub speed, and disturbance were examined and profoundly talked about.

Xun Sun, et al., (2017) [30] The introduction of a hurricane was generally assessed using the weight drop and the variety viability of the vicious breeze. In the present paper, a various-target progression of an extraordinary Stairmand tornado separator was executed using the response surface methodology (RSM), joined with computational fluid components (CFD) systems for restricting the weight drop and intensifying the combination adequacy. Ten brutal breeze geometrical components were considered in this work. Three of the mare analyzed using the RSM according to the outcomes of the previous screening tests. The second-demand response surface models for each response were adequately finished using the central composite structure (CCD) in the RSM. The alluring quality limit approach was used to streamline the geometrical components of the twister. Conversely with the reference model, the perfect twister model reductions the weight drop by $20.70 \%$ and the cut-off size by $75.38 \%$. The accuracy response of the surface models was certified and the precision of the perfect tornado model was furthermore endorsed using CFD; the results show awesome execution and constancy of the response surface model and the RSM upgrade result. The RSM and CFD techniques were used to effectively play out a tornado separator multi-target streamlining process, where the goals were characterized as minimizing the weight drop and amplifying the "all-out productivity". Ten geometrical variables were considered in the screening analyses, and three components, which effects affected the reactions, were applied in the RSM. The secondrequest reaction surface models for the weight drop and complete productivity were characterized

Typhoon separators are generally utilized in the gas strong partition forms. Their measurements and arrangements impact their exhibition and they have been generally contemplated in writing. In this exploration, the effects of utilizing a clay wear liner inside the mass of an uncommon residue authority typhoon, introduced in the Golgohar mining and mechanical organization in Iran, were examined [31-36]. In this exploration, some numerical figuring and programming investigation, given the CFD, has been completed and the outcomes have been contrasted and the aftereffects of the down to earth tests and recorded information. This examination shows the similarity of the aftereffects of the useful tests and the determined outcomes. In this examination, the stream was a two-stage, gas-strong, type and the impact of the strong materials, which are primarily iron material with 200 700-micron measurements, with the twister divider will build the disintegration. Along these lines, to lessen the disintegration an exceptional fired wear liner was utilized as a defensive layer for violent wind divider. Including this defensive layer has changed the geometry of the violent wind divider from various viewpoints; so the effects of this sort of liner on the exhibition and the division proficiency of the residue authority tornado ought to be researched. Thinking about the referenced clarifications and as indicated by the outline and the correlation between numerical outcomes embedded in table 2, the negative impact of this covering fired liner on division productivity was clear. As a recommendation for more research in the future one can take a shot at the advancement of geometric shape and other plan variables of this kind of liner. For instance, if liners are bent and finishing covering edges are considered such that no voids will be left after establishment, one can improve the twister productivity too forestall rough strong particles and residue enter the costly hardware like ESP and suction fan emplaced after the typhoon to diminish the significant expenses of these devices upkeep

Yaser H. Alahmadi, et al., (2016) [37] The paper explores the limited twirling stream in a twister. The numerical recreations are performed utilizing a proposed whirlpool thickness choppiness model, which represents the impacts of the streamline bend and revolution. This recognizes the present model from the traditional Eddy Viscosity Models (EVMs) that are known to neglect to foresee the Rankine vortex in whirling streams. Although computationally increasingly costly methodologies, the Reynolds Stress Model (RSM) and Large Eddy Simulation (LES), have exhibited a high ability to manage such streams these strategies are often unsuited for use in complex structure considers where computational speed and power are key variables. In the present approach, the Shear Stress Transport with Curvature Correction (SSTCC) disturbance model was changed by the presentation of the Richards on the number to represent the pivot and ebb and flow impacts. The numerical forecasts were approved utilizing exploratory outcomes and contrasted with the information obtained using the RSM model and different EVMs without the proposed modifications. The examinations began with a benchmark instance of a flowthrough a channel pipe with a U-turn, after which all the more testing re-enactments of a high whirling stream inside a typhoon separator gadget were performed. An alteration of the shear pressure transport model with bend amendment, known as SSTCC model Smirnov and Menter (2009), was proposed. The essential point of this work was to make another model that doesn't contain the complex Lagrangian subordinate term, which shows up in Eq. (35). This term was supplanted by the term speaking to the Richards on the number, which leads to a more straightforward turn/bend rectification technique. 


\section{International Journal of Engineering Applied Sciences and Technology, 2020 Vol. 4, Issue 11, ISSN No. 2455-2143, Pages 312-328 \\ Published Online March 2020 in IJEAST (http://www.ijeast.com)}

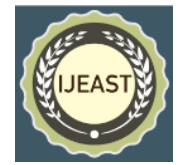

A.Y.T. Leung, et al., (2016) [38] Upgrade of fine particle (PM2.5) separation was huge for twister separators to diminish any additional purification technique required at the outlet. Right now, present test looks at was carried to examine the introduction of savage breeze separators adjusted with down-comer tubes at solid stacking rates from 0 to $8.0 \mathrm{~g} / \mathrm{m}^{3}$ with a 10 $\mathrm{m} / \mathrm{s}$ channel speed. The examination showed the practicality of down-comer tubes in diminishing the atom re-entrainment and extending the better separation with palatable pressure drops, which was enunciated at low solid stacking conditions. The preliminary outcomes were compared with the theories of Smolik and Muschelknautz. Theories were sufficient for explicit degrees, and theory breakdown was predominantly because of the disregard of molecule agglomeration, reentrainment and the reduction of twirling vitality, just as the expansion of divider grinding because of the essence of particles. The present examination demonstrated the noteworthy decrease of fine particle (PM2.5) reentrainment by down-comer tubes at the base of cyclone separators.

Xiaofeng Gu, et al., (2016) [39] To get further understanding into the dynamic property of the vortex, pressure signals at the different hub, spiral and circumferential situations in a gas-strong violent wind were estimated by multi-input dynamic weight transducer. By receiving likelihood thickness work, standard deviation, and force ghastly thickness techniques, the qualities of the weight signals were breaking down and talked about. Test results show that there are two prevailing frequencies (around $71 \mathrm{~Hz}$ and $179 \mathrm{~Hz}$ ) of the weight vacillation in the gas stream: the previous (around $71 \mathrm{~Hz}$ ) was identified with the semiconstrained vortex turn; the last mentioned (around 179 $\mathrm{Hz}$ ) was identified with the swing of the semiconstrained vortex centre. The molecule strands movement in the gas-strong stream decreases the scattering of weight change, mitigates the off-pivot wonder of the centre somewhat, and includes a prevailing low recurrence $(0.31 \mathrm{~Hz} \sim 0.86 \mathrm{~Hz})$ which gets higher with the expansion of channel solids stacking. The exploratory outcomes can give understanding into the weight change in a gas-strong violent wind separator. Weight signals at different situations in a gas-strong typhoon were estimated by multi-input dynamic weight transducer and broke down by a few techniques in this paper.

Aerogels might be orchestrated by removing the fluid dissolvable from an organogel utilizing supercritical $\mathrm{CO}_{2}$. The blend $\left(\mathrm{CO}_{2}\right.$-dissolvable) leaving the autoclave ought to be isolated and all the dissolvable in the example ought to be recuperated toward the finish of the extraction. These studies [40-47] manages a pilot-scale unit where $\mathrm{CO}_{2}$ and the dissolvable, here toluene, are isolated utilizing a course of twister separators. During the division, the toluene recuperation rate was underneath
$65 \%$. To decide the explanations for this breakdown, a thermodynamic report and a hydrodynamic report (Computational Fluid Dynamics, CFD) of the blend partition in the violent winds were completed. As per CFD, typhoons permit a productive mechanical division of the fluid dissolvable and the vaporous $\mathrm{CO}_{2}$.

Dheeraj Saini, et al., (2016) [48] Rajasthan and Gujarat on being situated at the tropical belt get nearly the most noteworthy sun-oriented illumination in India. Simultaneously, such parched desert areas are honored with dust. This vitality can be saddled utilizing, for example, sunlight based warm innovation. The recouped warmth can be utilized for applications, similar to, heat treatment of metal. For this reason, an open volumetric air beneficiary-based sun-based pinnacle innovation idea was received. In this beneficiary, concentrated sunoriented light was engaged onto permeable safeguard, which was available to air. The air was utilized as warmth move liquid with suction. The suspended residue particles in the air will store in the pores of the open safeguard and even go to the internals of the total frameworks. This is relied upon to harm the collector and forestall persistent activity of such a framework. In this paper, the structure and assessment of a tornado separator for cleaning and assortment procedure of residue from open volumetric air beneficiary were portrayed. In this paper plan and assessment of tornado separator, which was required for cleaning an assortment of residue for open volumetric air beneficiary was introduced. This was essential for the activity of open volumetric air beneficiary-based sun-powered warm innovation in bone-dry desert areas, as, Rajasthan. The plan of tornado separator depends on the ideal proficiency for a molecule size with a given air mass stream rate. The activity of this framework will rely upon the necessary force, which relies upon pressure-drop over the structured tornado separator.

Jianfei Song, et al., (2016) [49] In light of the mechanical foundation of a carbonaceous affidavit in optional typhoon separators of FCC units, this paper centres around molecule testimony example and examination of elements on the statement on the external surface of vortex discoverer of an auxiliary violent wind. Molecule statement tests were completed with a 500mmwidth twister in a cool model pilot test stage. Testimony designs were gotten under various gulf gas speeds, molecule fixations and trial materials and so forth. Moreover, the discrete stage model (DPM) was applied to get particle conveyance in the annular space and the impact of molecule properties and operational conditions on the mass of stored particles was additionally explored. Moreover, the Gas protecting strategy was, to begin with, investigated to stay away from the molecule affidavit. The outcomes will give test proof to breaking down the testimony procedure and help to additionally comprehend the affidavit instrument. Molecule affidavit design on the external surface of vortex discoverer of typhoon separator with the barrel breadth $500 \mathrm{~mm}$ and factors on the testimony were tentatively explored. The conveyance 


\section{International Journal of Engineering Applied Sciences and Technology, 2020 Vol. 4, Issue 11, ISSN No. 2455-2143, Pages 312-328 \\ Published Online March 2020 in IJEAST (http://www.ijeast.com)}

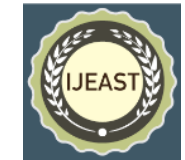

of particles in the annular space and their attachment superficially was additionally numerically contemplated. A starter study on dodging particles affidavit was tentatively completed.

Le Van Sy, (2016) [50] Gas-Liquid Cylindrical Cyclone Separator (GLCC) was broadly utilized in the oil business with potential field applications. Its presentation was emphatically impacted by the gulf design. The 27-degree ideal slanted bay edge has been tentatively watched for GLCC with a similar distance across of body and delta. For different GLCCs, the impact of delta points on stream design and their exhibitions have not been examined. The fundamental objective of the present examination was to profoundly comprehend the progressions of stream design as for various slanted points and stream conditions. Twelve GLCCs with various slanted points were numerically researched by utilizing the Reynold Stress choppiness model to foresee the stream design with GLCC. The appropriation of outspread, pivotal and digressive speed profiles and their greatest sizes as for the difference in bay point were deliberately considered in this examination. In this paper, a numerical re-enactment (CFD) in blend with reasonable investigations has been utilized as a potential instrument that can assist with bettering comprehend the impact of slanted gulf edge on a mind-boggling stream example of the GLCC separators in which have little breadth proportion of the body and bay.

Outflow reduction in violent wind execution was improved by expanding assortment adequacy or diminishing vitality utilization. The object of the examination [51-57] was to measure the weight drop and fine particulate (PM2.5) assortment of 1D3D twisters $(\mathrm{H}$ $=4 \mathrm{Dc}, \mathrm{h}=1 \mathrm{Dc})$ at gulf speeds from 8 to $18 \mathrm{~m} \mathrm{~s}^{-1}(\mathrm{Stk}=$ 0.7-1.5) utilizing heterogeneous particulate as a test material at bay fixations from 3 to $75 \mathrm{gm}-3$. Tornado exhaust was gone through channels. Laser diffraction molecule size appropriation examination was utilized to appraise PM2.5 discharges. Reaction surface models indicated a solid connection between violent wind pressure misfortune (Euler number) and delta speed and anticipated a $46 \%$ decrease in pressure misfortune for a $25 \%$ decrease in bay speed (Stokes number). The model for PM2.5 discharges was less conclusive and, shockingly, anticipated a $31 \%$ lessening in PM2.5 outflows while working $25 \%$ beneath the structure bay speed. Working underneath the structure delta speed (at a lower Stokes number) to lessen pressure misfortunes (Euler number) would decrease both the money related and the ecological expense of acquiring power. The surprising co-advantage proposed by these preliminaries was that outflow reduction may improve simultaneously, however other exact preliminaries have demonstrated emanations to be autonomous of channel speed and Stokes Number Cyclones were tried to all the more likely comprehend the impacts of bay speed and gulf fixation on pressure drop and discharges. Changed 1D3D violent winds were worked at channel speeds from about half to marginally more than structure, from 8 to $18 \mathrm{~ms}^{-1}$, over a scope of gulf fixations from 3 to $75 \mathrm{~g} \mathrm{~m}-3$. Tornado pressure drop, $\Delta \mathrm{P}$, was estimated legitimately. Radiated absolute mass gathered on channels and molecule size circulation investigation was utilized to evaluate PM2.5 discharges, introduced in this paper as a mass of PM2.5 for every $\mathrm{kg}$ of complete material sustained to the violent wind.

Pranas Baltrenas, et al., (2015) [58] Twister Dust Separators are gadgets frequently used to channel strong particles from pipe gas. Such violent winds should channel however much particulate issue from the vaporous stream as could be expected. Simultaneously, they should just acquaint negligible weight misfortune with the framework. Subsequently, assortment effectiveness must be augmented and pressure misfortune limited. Both the assortment effectiveness and weight misfortune are vigorously impacted by twister geometry. In this paper, we streamline the inward geometrical development of multichannel tornado to up cleaning productivity parameter. This examination shows how geometry includes cleaning productivity. At wind, current rates from $12 \mathrm{~m} / \mathrm{s}$ to $18 \mathrm{~m} / \mathrm{s}$ after looks into the ideal cleaning productivity accomplished in the threelevel typhoon at $16 \mathrm{~m} / \mathrm{s}$ normal wind stream rate in tornado channels when the treated air was contaminated with 20-micrometer size rock particles and stream circulation proportion was 75/25. For low thickness particles (wood debris) for the 20-micrometer width top productivity built up in 6-channel twister at the $16 \mathrm{~m} / \mathrm{s}$ wind current rate in the channels and stream dissemination proportion $75 / 25$.

Xiaoming Luo, et al., (2015) [59] The client characterized work of Fluent test system was received to characterize introductory states of transient slug stream, and a re-enactment strategy, which depended on VOF multi-stream model, of slug stream was created to lead numerical reproduction to explore on slug scattering in channel rectifier funnel of tube-shaped tornado separator. Stream parameters of introductory bay slug were given by building up a one-dimensional relentless slug stream model. This paper reproduced the dissemination procedure of hydrodynamic slug in rectifier pipes, where the slug dispersed under the co effect of measurement extending pipes and descending slanted channels. The divergent power field in descending slanted helical funnels guaranteed the tendency and security of the gasfluid interface at the equivalent time with the assistance of the client characterized work of Fluent test system, this paper embraced the one-dimensional relentless slug stream model to characterize beginning states of transient slug stream, and the scattering law of rectifier pipes was all around re-enacted dependent on the VOF model. The reproductions in this paper not just concentrated the streaming law of delta rectifier channels of GLCC, yet additionally investigated the techniques to re-enact the slug stream. 


\section{International Journal of Engineering Applied Sciences and Technology, 2020 Vol. 4, Issue 11, ISSN No. 2455-2143, Pages 312-328 \\ Published Online March 2020 in IJEAST (http://www.ijeast.com)}

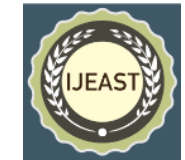

L.S. Brar, et al., (2015) [60] The present work deals with the examination of fluctuating estimations on the presentation of the gas savage breezes. Three remarkable separations across viz. 0.8D, 1.0D, and 1.2D had been considered ( $\mathrm{D}$ being the standard estimation of the storm and the constants were the scaling factors) and their ramifications for the weight drop, collection capability, and cut-off separation across had been evaluated. NavierStokes conditions were discretized using constrained volume techniques which were lit up iteratively with mechanically open CFD code FLUENT 6.3.2 on square unstructured hexahedral work. Reynolds stress model (RSM) had been used as an end model for Reynolds found the centre estimation of Navier-Stokes conditions, which enlightens for pressure tensors together with the condition for the spread rates. It had been found that extension in brutal breeze width grows the arrangement capability to the drawback of extending pressure drops over the gas storms. Numerical re-enactments on all the tornado models with expanding twister principle body distance across have been completed effectively utilizing the RSM choppiness model. It was seen that this fierce model was skilled to determine the twofold vortex type stream with a significant level of precision.

Lu Duan, et al., (2015) [61] The technique for entropy age examination was applied to look at the impacts of the leave pipe distance across and the bay measurements on the entropy age in tornados. The stream fields of the typhoons were reproduced utilizing the Reynolds Stress Model (RSM). The re-enactment results were utilized to ascertain three sorts of entropy age brought about by direct dissipation, turbulent dissemination, and divider grating and afterward to decide the exergy loss. The results proposed that the exergy misfortune in tornados increments with the bay speed and that it diminishes with the leave pipe distance across and the delta dimensional parameter KA. What's more, the vitality utilization in the violent winds mostly came about because of the tempestuous dispersal and the divider erosion. The districts close to the vortex discoverer and the passageway of the residue container are the principle vitality utilization areas in the cyclone. The level of the entropy age around the vortex discoverer first declines and afterward increments with the expansion of the leave pipe width and KA, which was the contrary conduct to that around the passage of the residue container. Recreations were performed for typhoons of various gas leave pipe breadths and delta measurements. The recreation results were contrasted and the distributed outcomes and the test information. The entropy age examination and exergy investigation were conducted based on the recreation results.

Andrew Y.T. Leung, et al., (2015) [62] The present paper relies upon CFD showing of gas-solid stream in tropical storm separators with different build-up outlet geometries (with and without down-comer tubes at the brutal breeze base) to analyze the stream traits and the twister execution. Numerically procured twister execution parameters, collection capability, and weight drop were differentiated and preliminary outcomes. Changes in the atom headings as a result of the assortment of the stream field inside the storm separators with the effect of the build-up outlet section were also researched in detail. The proliferation results, as it were, agreed with the exploratory results, and blunders were realized by exhibiting requirements and abnormalities of the particle following model with the real gas-solid stream. Nevertheless, indistinguishable from the preliminaries, the CFD amusements furthermore have foreseen the increases in grouping efficiencies by dust outlet geometries balanced with down-comers. Inside satisfactory weight drops LES based CFD displaying of tornado separator gas strong stream was led for five twister geometries with various residue outlet geometries to dissect the stream field and typhoon execution with down-comer tubes at the violent wind base. The typhoon execution parameters, assortment productivity, and weight drop were contrasted with the exploratory outcomes.

The attributes of a molecule separator were numerically researched utilizing the idea of a uniflow violent wind. The target of the study was to anticipate the inside stream field and to examine the impact of stream streams on molecule development in a uniflow typhoon separator. The movement of strong particles in a stream field was reproduced utilizing the Eulerian-Lagrangian approach [63-69]. Gulf temperature (Tin = 300-1100 K) and weight (Pin= 1-9 bar) were shifted for the underlying conditions. Residue gas blends (i.e., molecule loaded stream) were infused into the separator channel at $\mathrm{u}_{\mathrm{in}}=3-15 \mathrm{~m} / \mathrm{s}$. Estimation results demonstrated that the Eulerian-Lagrangian approach was valuable for adjusting the two-staged gooey choppiness stream. The distribution zone was anticipated under a vortex discoverer, while a helical stream created in the bearer gas outlet. Partition effectiveness diminished with an expansion in the dustgas temperature and pressure and expanded with an increment in molecule stacking, gulf speed, and molecule diameter Numerical reproduction was utilized to explore an inward stream field and to foresee molecule detachment productivity of a UCS with a CFD program (ANSYS Fluent ver. 12.01). The structure of the UCS was described by the age of a solid twirl stream in the separator channel and a uniflow stream of a residue gas blend (i.e., molecule loaded stream) in the separator body.

S.B. Kuang, et al., (2015) [70] Utilization of huge thick medium twisters (DMCs) was a potential pattern in the coal business yet its use was restricted by the poor division productivity of fine particles. This paper exhibits a numerical investigation of the multiphase streams and execution of DMCs utilized for coal readiness by methods for the two-liquid model. The legitimacy of the model has been confirmed by different applications. It was utilized here to examine the practices of fine particles in a 2-m DMC that was bigger than the greatest DMCs revealed up to this point in the coal 


\section{International Journal of Engineering Applied Sciences and Technology, 2020 Vol. 4, Issue 11, ISSN No. 2455-2143, Pages 312-328 \\ Published Online March 2020 in IJEAST (http://www.ijeast.com)}

business. The numerical outcomes show that in the extraenormous DMC, the poor partition proficiency of fine particles deteriorates contrasted with that of a generally utilized 1-m DMC. This inadequacy was seen as credited to the solid vortexes created and the uneven partition zone that can be described by the relationship between weight inclination and digressive speed. A few alterations regarding mounting degree, operational Head, cone-like area length, and gulf number are acquainted with improving the exhibition of the 2-m DMC. It was demonstrated that the detachment productivity of fine particles in the 2-m DMC can be better contrasted with the 1-m DMC by expanding the Head or funnel-shaped segment length because such adjustments decrease the deviated partition zone or potentially measure of vortexes. Enormous DMCs can offer various advantages yet experience the ill effects of crumbled partition proficiency of fine particles. The exhibition of an extraenormous DMC, whose body breadth was past the range detailed previously, has been examined utilizing the as of late created two-liquid model, with uncommon reference to the practices of fine particles

Rainier Hreiz, et al., (2014) [71] This paper comprises a test investigation of the division exhibitions of a gas-fluid round and hollow tornado (GLCC) separator that premiums the oil business. The worldwide hydrodynamics conduct in the GLCC was portrayed by stream representation under different inflow working conditions. The impact of the gulf spout structure on the exhibitions of the separator was examined by utilizing three unique spouts, and it ends up being a key parameter. With an inadequate spout limitation, low whirl power was granted to the stream. Because of lacking radiating impacts, fluid was rashly persisted by the gas as flooding happens in the separator upper part. High measures of gas are likewise conveyed under by the fluid stream. Then again, with a too serious spout assembly, the significant drag applied by the gas prompts fluid "short-circuiting" the typhoon toward the gas outlet. Notwithstanding the spout structure, the separator exhibitions are impacted by marvels, for example, fluid connecting or the event of the slug stream system at the violent wind channel. This paper prompts a superior comprehension of the connections between the hydrodynamics in the GLCC and its operational points of confinement, which was important to empower dependable scaling up devices. Right now, the impact of the channel spout structure on the exhibitions of a gasfluid round and hollow typhoon (the GLCC) working in a full separator setup has been examined tentatively. Working the typhoon at the skirt or more its operational cut off points was useful to distinguish diverse key components that utmost the scope of the normal working of the framework.

VladasVekteris, et al., (2014) [72] This paper presents wind stream speed profiles acquired in regular and acoustic tornado separators. It was demonstrated that the vortex wind stream was made in acoustical typhoon separator in the nearness of optional counter-current wind stream. It was acquired that in acoustic tornado separator gaseous tension heartbeats happen at a recurrence of $8 \mathrm{kHz}$ and weight sufficiency arrives at an estimation of 170 decibel. Partition productivity of acoustic typhoon separator was set up tentatively. The most extreme stream speeds were seen at the fringe of the vortex. Stream speed firmly diminishes when moving toward the pivot of tornado separator. The spiral stream vanishes and the hub shows up close to the hub of typhoon separator when the span turns out to be under 30 mm.

Sujeet Kumar Shukla, et al., [73] The effect of showing of speed changes on the desire for variety adequacy of tornado separators had been numerically investigated using the Reynolds stress roughness model (RSTM) and enormous vortex generation (LES). The EulerianLagrangian exhibiting approach of CFD code Fluent 6.3.26 has been used to re-enact the three-dimensional, insecure tempestuous gas-strong streams in a Stairmand high effectiveness violent wind. The recreated outcomes have been contrasted and test perceptions accessible in the writing. The examination of results shows that the RSTM and the LES had enough foreseen the mean stream field. Outcomes of the present examination show that the LES has extraordinary execution on the desire for fluctuating stream field and variety capability for each atom size. In any case, the exhibition of the RSTM was discovered poor as far as expectation of speed variances and assortment effectiveness, particularly for little particles. This identifies with the processing of the vortex centre wonder, which was settled all the more precisely by LES when contrasted with the RSTM reenactment. The outcomes propose that the expectation of assortment proficiency, particularly for little particles was extraordinarily affected by the recreation of speed vacillations in typhoons. A numerical methodology utilizing the Reynolds stress choppiness model (RSTM) and enormous whirlpool re-enactment (LES) for disturbance terminations were utilized to contemplate the impact of demonstrating of speed changes on the forecast of assortment proficiency of violent winds. The outcomes anticipated utilizing the Eulerian-Lagrangian approach of CFD code Fluent 6.3.26 were approved against the test esteems given in writing.

Lian-ping Dong, et al., (2013) [74] In light of the water-just twister, another violent wind named tornado section separator (CCS) was created by joining a positive cone with the regular negative cone to frame a non-consistent three-arrange muddled cone (CC). The presentation of the CCS was contrasted and that of waterjust tornados with the single edge of $70^{\circ}, 90^{\circ}, 110^{\circ}$ and $120^{\circ}$ by coal ooze division tests. Contrasted and the water-just typhoons with single cone structure, the partition execution of the CCS with CC structure was essentially improved and substantially fewer misfortunes of $+0.25 \mathrm{~mm}$ low thickness coarse coal were come about. It delivered a likely blunder of 0.058 for $+1 \mathrm{~mm}$ divisions, and 0.083 for $0.5-1 \mathrm{~mm}$ portion. It additionally 


\section{International Journal of Engineering Applied Sciences and Technology, 2020 Vol. 4, Issue 11, ISSN No. 2455-2143, Pages 312-328 \\ Published Online March 2020 in IJEAST (http://www.ijeast.com)}

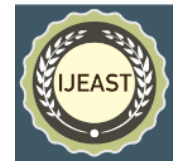

gave a flaw of 0.202 for a fairly more extensive size scope of $+0.25 \mathrm{~mm}$. All outcomes show that the CCS was a coarse coal partition hardware of high productivity Based on the water-just violent wind, another gadget named CCS was created by incorporating a positive cone with a normal negative cone of $90^{\circ}$. The epic cone structure was an entangled three-arrange spasmodic one. The partition results show that the CCS improved the division of coarse coal and introduces the most reduced plausible mistake and least flaw as contrasted and the conventional water-just violent winds with single cone. The plausible mistake for the wide size part of $+0.25 \mathrm{~mm}$ was 0.130 and the blemish for coal detachment was as low as 0.202 .

Cuizhi Gao, et al., (2013) [75] The effect of tornado estimations on parcel execution was important. A perfect twister stature, assumed the most outrageous viability rough wind height here, can make the efficiency of storm separator best. At present, the effect of channel estimations on the best efficiency rough wind stature was considered through investigations and CFD reenactments. The exploratory results show that the best efficiency twister height diminishes with the lessening of the delta estimations. CFD multiplications suggest the trademark vortex length curtails with the reduction of the inlet estimations. There was an essential consistency between the changing examples of the most outrageous adequacy twister stature and the trademark vortex length. Moreover, the extending of the cone height accomplishes the increase of customary vortex length and results in the development of capability. Regardless, when the circumstance of the vortex end goes into the space of the cone, the parcel capability goes to lessen. This wonder exists in a wide scope of hurricanes with different delta estimations. It suggests that the typical vortex length expect a colossal activity in choosing the most outrageous capability fierce breeze height, and the effect of the straight estimations on the best profitability tornado stature might be credited with the effect of the bay estimations on the regular vortex length. The partition effectiveness improves with expanding the cone tallness. In any case, the greatest proficiency stature exists, and it was essentially impacted by the bay measurements. Trial results demonstrate that with the abatement of the bay measurements, the greatest productivity tornado stature lessens. Although this pattern can be anticipated by current hypothetical models of the most extreme effectiveness typhoon stature, there are noteworthy contrasts between the test information and the hypothetical outcomes. CFD recreations recommend the characteristic vortex length abbreviates with the decrease of the channel measurements.

\section{B. Findings in Cyclone Separator}

1. By analysing various parameters such as total height, inlet height, inlet width, inlet diameter which are the main geometry to reduce the pressure drop and increasing the collection efficiency, we came to know that the collection efficiency is affected by a cyclone separator.

2. By reducing the cone size result increases in efficiency without causing any changes to pressure drop of the cyclone separator, we came to know that the flow rate causes a high impact on the efficiency value.

3. The performance of cyclone separator is improved by minimizing energy consumption and maximizing collection efficiency.

4. By experiments and CFD simulations, we came to know that there is more consistency between cyclone height and vortex length in determining final efficiency value.

5. Through Eulerian-Lagrangian based numerical technique, we came to know that the pressure drop and collection efficiency will be reduced when increasing the exit diameter of the pipe.

6. Through CFD simulation of different exit diameters, we came to know that if the diameter of the exit tube increases, the pressure drop will get reduced and a high collection of minute dust particles can be obtained.

7. By understanding several journals had discussed the effect of vortex finder diameter which creates an impact on cyclone performance. They studied the effect of vortex finder length on separation, efficiency, pressure drop, and swirl flow. These studies showed that separator performance can be improved by optimizing the size of the vortex finder.

8. By performing comparative observation of cyclone models using response surface methodology (RSM), backpropagation neural network (BPNN), and group method of facts handling (GMDH) networks to develop top of the line predictive cyclone models and also carried out multi-objective optimization for larger models and minimizing version, the usage of genetic algorithm (GA), CFD became performable in preference to experimental method to get the anticipated values for modelling of strain drop and efficiency.

9. The separation efficiency is increased and then decreased with an increase of inlet angle. The maximum separation efficiency is attained at the inlet angle of $15 \mathrm{deg}$. The separation efficiency is increased when the diameter of the particle increases. The pressure drop decreases at first and then increases with the increase of the inlet angle. Gas entering the cyclone at an inlet angle of $0 \mathrm{deg}$ has the minimum pressure drop, and the variation range of pressure drop at a negative inlet angle is larger than that of a positive inlet angle. 


\section{International Journal of Engineering Applied Sciences and Technology, 2020 Vol. 4, Issue 11, ISSN No. 2455-2143, Pages 312-328 \\ Published Online March 2020 in IJEAST (http://www.ijeast.com)}

10. To find out the collection performance of a cyclone, the term "total efficiency", does not only act meaningfully for experiments, but the optimization of a cyclone using CFD approach also gives the valid data about the process. In comparison, decreasing the inlet height and inlet width and decreasing the vortex finder diameter was a much more efficient way to increase the collection efficiency, also resulting in a favorable lower pressure drop.

\section{CONCLUSION}

The study from several journals gave a clear view of the geometrical parameters of the cyclone separator and how it creates an impact on the performance of achieving higher efficiency for different denser particles. The most important parameters to look while designing the cyclone separator are inlet height, inlet width, outlet diameter, cone diameter, and total height. Increasing the flow rate and inlet velocity leads to a greater number of particle collections as long as the pressure drop is not high. Pressure drop is one of the most important factors for attaining the highest efficiency. When the pressure drop gets reduced, the efficiency gets increases. Thus, all the cyclone separators were designed to maximize the efficiency for minute size particles and to get the highest cut off diameter.

\section{ACKNOWLEDGEMENT}

We wish to express our sincere thanks to our project guide, Dr. R. Suresh Kumar for guiding us in a right path by motivating and clearing our doubts related to our project/review and also to our faculties who empowered our knowledge.

\section{REFERENCES}

[1] Jun-Hyung Lim, Su-In Park, Heun-Jae Lee, Muhammad Zeeshan Zahir, Se-JinYook (2020). 'Performance evaluation of a tangential cyclone separator with additional inlets on the cone section' Powder Technology 359 (2020) 118-125.

[2] Li Qiang, Wang Qinggong, Xu Weiwei, Zhu Zilin, Zhu Konghao (2019). 'Experimental and computational analysis of a cyclone separator with a novel vortex Finder' - Powder Technology PII: S0032-5910(19)30896-4.

[3] Yanqin Mao, Wenhao Pu, Hao Zhang, Qiyu Zhang, Zhangyang Song, Kaiqi Chen, Dong Han (2019). 'Orthogonal experimental design of an axial flow cyclone separator' - Chemical Engineering \& Processing: Process Intensification144 (2019) 107645.

[4] Mofarrah Masoumeh, Li Shuran, Zheng Qinzhen, Deng Guanle, Liu Zhen, Yan Keping (2019).
'Performance evaluation of a new micro gas cyclone using simulation and experimental studies to capture indoor fine particles' - Advanced Powder Technology 30 (2019) 1151-1159.

[5] Donggeun Park, Jemyung Cha, Moonjeong Kim \&Jeung Sang Go (2019). 'Multi-objective optimization and comparison of surrogate models for separation performances of cyclone separator based on CFD, RSM, GMDH neural network, back propagation-ANN and genetic algorithm' Engineering Applications of Computational Fluid Mechanics2019, VOL. 14, NO. 1, 180-201.

[6] Omid Reza Nassaj, DavoodToghraie, Masoud Afrand, (2019). 'Effects of multi inlet guide channels on the performance of a cyclone separator' - Powder Technology 356 (2019) 353-372.

[7] R. S.Kumar, J. Alexis \&V. S. Thangarasu, (2017). Optimization of high-speed $\mathrm{CNC}$ end milling process of BSL 168 Aluminium composite for aeronautical applications. Transactions of the Canadian Society for Mechanical Engineering, 41(4), 609-625

[8] S. R. Kumar, J. S. Alexis \&V. S. Thangarasu, (2017). Experimental Investigation of Influential Parameters in High-Speed Machining of AMS 4205. Asian Journal of Research in Social Sciences and Humanities, 7(2), 508-523.

[9] S. Ganeshkumar, V.Thirunavukkarasu, R. Sureshkumar, S. Venkatesh \&T. Ramakrishnan.Investigation of Wear Behaviour of Silicon Carbide Tool Inserts and Titanium Nitride Coated Tool Inserts in Machining of EN8 Steel.

[10]S. Kumar, J. Alexis \&V. S. Thangarasu (2016). Prediction of machining parameters for A91060 in end milling. Advances in Natural and Applied Sciences, 10(6 SE), 157-164.

[11]R. S. Kumar, V. S. Thangarasu\& S. J.Alexis(2016). Adaptive control systems in CNC machining processes--a review. Advances in Natural and Applied Sciences, 10(6 SE), 120-130.

[12] S. Kumar, J. Alexis\&K. P. Dhanabalakrishnan (2015). Application of GA \& ANN for the optimization of cutting parameters for end milling operation- A comparison. International Journal of Applied Engineering Research, 10(20), 1809218107.

[13] Wei Zhang, LinlinZhanga, Jingxuan Yang, Xiaogang Hao, Guoqing Guan, ZhihuaGaoc (2019). 'An experimental modeling of cyclone separator efficiency with PCA-PSO-SVR algorithm - Powder Technology 347 (2019) 114-124. 


\section{International Journal of Engineering Applied Sciences and Technology, 2020 \\ Vol. 4, Issue 11, ISSN No. 2455-2143, Pages 312-328 \\ Published Online March 2020 in IJEAST (http://www.ijeast.com)}

[14] NikolayMikheev, IlyaSaushin, AnnaGoltsman, VictorFafurin (2018). 'Data of numerical simulation and experimental research on the design of a cyclone separator with a high flux density' - Data in Brief 20(2018)1836-1843.

[15] Nikolay Mikheev, Ilya Saushin, Anton Paereliy, Dmitry Kratirov,Kirill Levin (2018). 'Cyclone separator for gas-liquid mixture with high flux density' - Powder Technology PII: S00325910(18)30670-3.

[16] Seung-Yoon Noh, Ji-EunHeo, Sang-Hee Woo, SangJun Kim, Myeong-HakOck, Young-Jin Kim, SeJinYook (2018).' Performance improvement of a cyclone separator using multiple subsidiary cyclones - Powder Technology PII: S0032-5910(18)30507-2.

[17] S.Venkatesh,

M.Sakthivel, S.Sudhagar\&S.AjithArulDaniel (2018). 'Modification of the cyclone separator geometry for improving the performance using Taguchi and CFD approach' - Particulate Science and Technology ISSN: 0272-6351.

[18] Shuyan Wang, Haolong Li, Ruichen Wang, Xu Wang, Ruichao Tian, Qiji Sun (2018). 'Effect of the inlet angle on the performance of a cyclone separator using5 CFD-DEM' - Advanced Powder Technology $\mathrm{xxx}(2018) \mathrm{xxx}-\mathrm{xxx}$.

[19]Bingtao Zhao, YaxinSu (2018). 'Particle size cut performance of aerodynamic cyclone separators: Generalized modeling and characterization by correlating global cyclone dimensions' - Journal of Aerosol Science.

[20]T. Ramakrishnan, \&S. Pavayee Subramani (2018). Investigation of Physico-Mechanical and Moisture Absorption Characteristics of Raw and Alkali Treated New Agave Angustifolia Marginata (AAM) Fiber. Materials Science, 24(1), 53-58. [SCI \& Scopus IF: 0.593]

[21]T. Ramakrishnan \&P. S. Sampath (2017). Dry Sliding Wear Characteristics of New Short Agave Angustifolia Marginata (AAM) Fiber-Reinforced Polymer Matrix Composite Material. Journal of Biobased Materials and Bioenergy, 11(5), 391-399. [Scopus \& SCI, IF: 2.993]

[22] R. Jeyakumar, P.S. Sampath, R.Ramamoorthi\&T. Ramakrishnan(2017). Structural, morphological and mechanical behavior of glass fiber reinforced epoxy nanoclay composites. The International Journal of Advanced Manufacturing Technology, 93(1-4), 527535. [Scopus \& SCI, IF: 2.663]
[23]T. Ramakrishnan \&P. S. Sampath, (2017). Experimental investigation of mechanical properties of untreated new Agave Angustifolia Marginatafiber-reinforced epoxy polymer matrix composite material. Journal of Advances in Chemistry, 13(4), 6120-6126. [Scopus \& IF: 1.131]

[24]R. Ramamoorthi, R. Jeyakumar\&T. Ramakrishnan (2017). Effect of Nanoparticles on the Improvement of Mechanical Properties of Epoxy Based Fiber Reinforced Composites - A Review. International Journal for Science and Advance Research in Technology, 3(11), 1251- 1256.

[25] JirawatJuengcharoensukying, KunakornPoochinda, BenjaponChalermsinsuwan (2017). 'Effects of Cyclone Vortex Finder and Inlet Angle on Solid Separation Using CFD Simulation' - Science Direct 138 (2017) 1116-1121.

[26] Adi Surjosatyo, Adi Respati, HafifDafiqurrohman, Muammar (2017). 'Analysis of the Influence of Vortexbinder Dimension on Cyclone Separator Performance in Biomass Gasification System' ScienceDirect 170 (2017) $154-161$.

[27]An-Ni Huang, Norio Maeda, Daiki Shibata, Tomonori Fukasawa, Hideto Yoshida, Hsiu-Po Kuo, Kunihiro Fukui (2017). 'Influence of a Laminarizer at the Inlet on the Classification Performance of a Cyclone Separator' - Separation and Purification Technology PII: S1383-5866(16)31594-5.

[28] DzmitryMisiulia, KhairyElsayed, Anders Gustav Andersson (2017). 'Geometry optimization of a deswirler for cyclone separator in terms of pressure drop using CFD and artificial neural network' Separation and Purification Technology PII: S13835866(16)32613-2.

[29] Hamed Safikhani, Javid Zamani, Mohammadreza Musa (2017). 'Numerical study of the flow field in new design cyclone separators with one, 5 two and three tangential inlets' - Advanced Powder Technology xxx (2017) xxx-xxx.

[30]Xun Sun, Sung Kima, Seung Deok Yang, Hyun Soo Kim, Joon Yong Yoon (2017). 'Multi-objective optimization of a Stairmand cyclone separator using response surface methodology and computational fluid dynamics' - Powder Technology320 (2017) 51-65.

[31] Mohsen Khorasanimohammadrezaei, Nasrin Aminizadeh (2016). 'The Impact of a ceramic wear liner on the separation efficiency of a particular cyclone dust collector' - ScienceDirect157 (2016) $414-421$ 


\section{International Journal of Engineering Applied Sciences and Technology, 2020 Vol. 4, Issue 11, ISSN No. 2455-2143, Pages 312-328 \\ Published Online March 2020 in IJEAST (http://www.ijeast.com)}

[32] T. Ramakrishnan, P. S. Sampath \&R. Ramamoorthi (2016). Investigation of Mechanical Properties and Morphological Study of the Alkali Treated Agave Angustifolia MarginataFiber Reinforced Epoxy Polymer Composites. Asian Journal of Research in Social Sciences and Humanities, 6(9), 461-472.

[33]T. Ramakrishnan\& P. S. Sampath(2016). Thermogravimetric Analysis (TGA) and the Effect of Moisture Absorption on the Mechanical Properties of New Agave Angustifolia Marginata 3 Fiber (AAMF) Reinforced Epoxy Polymer Composite Material, International Journal of Printing, Packaging \& Allied Sciences, 4(5), 32453256. [Global IF: 0.12]

[34]T. Ramakrishnan, K. Sathish, P. S. Sampath \&S. Anandkumar(2016). Experimental investigation and optimization of surface roughness of AISI 52100 alloy steel material by using the Taguchi method. Advances in Natural and Applied Sciences, 10(6 SE), 130-138.

[35] K. Sathish, T. Ramakrishnan \&S. Sathishkumar (2016). Optimization of turning parameters to improve the surface finish of $16 \mathrm{Mn} \mathrm{Cr} 5$ material. Advances in Natural and Applied Sciences, 10(6 SE), 151-157.

[36]S. Karthik Raja S.Balasubramani, S.Venkatesh, T.Ramakrishnan (2015). Effect of Cryogenic Tempering on Steel, International Journal of Mechanical and Civil Engineering, 2 (6), 98-113.

[37] Yaser H. Alahmadi, Andrzej F. Nowakowski (2016). 'Modified shear stress transport model with curvature correction for the prediction of swirling flow in a cyclone separator' - Chemical Engineering Science 147 (2016) 150-165.

[38] Sakura GanegamaBogodage, A.Y.T. Leung (2016). 'Improvements of the cyclone separator performance by down-comer tubes' - Journal of Hazardous Materials311 (2016) 100-114.

[39]Xiaofeng Gu, Jianfei Song, YaodongWei (2016). 'Experimental study of pressure fluctuation in a gassolid cyclone separator' - Powder Technology PII: S0032-5910(16)30261-3.

[40] MounaLazragDeisy Lizeth Mejia-Mendez C'ecile Lemaitre Philippe Hugh Emmanuel Stafford Rainier Hreiz Romain Privat Ahmed Hannachi Danielle Barth (2016). 'Thermodynamic and hydrodynamics study of gas-liquid flow in a cyclone separator downstream supercritical drying' J. of Supercritical Fluids PII: S0896-8446(16)30223-6.

[41]S. Venkatesh \& M. Sakthivel (2017). 'Numerical Investigation and Optimization for Performance Analysis in Venturi Inlet Cyclone Separator',
Desalination and Water Treatment, Vol. 90, No. 9, pp. 168-179. [Desalination publication, Impact Factor: 1.631, Scopus].

[42] S. Venkatesh, M. Sakthivel, S.Sudhagar\& S. Ajith Arul Daniel(2018). 'Modification of the cyclone separator geometry for improving the performance using Taguchi and CFD approach', Particulate Science and Technology, Doi:10.1080/02726351.2018.1458354. [Taylor \& Francis publication, Impact Factor: 0.785, Scopus].

[43] S. Venkatesh, I. Bruno Clement, M.Avinasilingam,\& E. Arulkumar (2017). "Design of Experiment Technique for Improving the Performance of Stirling Engine", International Research Journal of Engineering and Technology, Vol. 4, No. 5, pp. 6265.

[44] S. Venkatesh, S.Balasubramani, S.Venkatramanan\& L. Gokulraj. "Standardization of hpx spool for lead time reduction of string test", Journal of Mechanical and Civil Engineering, Vol. 2, No. 6, pp. 62-79.

[45] S. Kousalya Devi, S. Venkatesh \&P.Chandrasekaran. (2015). "Performance Improvement of Venturi Wet Scrubber, "Journal of Mechanical and Civil Engineering, Vol. 2, No. 4, pp. 1-9.

[46] P.Arunkumar, P.Dhachinamoorthi, K.Saravanakumar\&S. Venkatesh (2014). "Analysis and Investigation of Centrifugal Pump Impellers Using CFD," Engineering Science and Technology: An International Journal, Vol. 4, No. 4, pp. 112-117.

[47]K. P. Dhanabalakrishnan, J. Abuthakir, R. Subramanian, S. Venkatesh (2015). "Evaluation of Tensile Properties of Particulate Reinforced AlMetal Matrix Composites," Engineering Science and Technology: An International Journal, Vol. 5, No. 1, pp. 173-175.

[48] Gurveer Singh, Dheeraj Saini, Laltu Chandra (2016).' On the evaluation of a cyclone separator for cleaning of open volumetric air receiver' - Applied Thermal Engineering PII: S1359-4311(15)01134-5.

[49] Jianfei Song, Yaodong Wei, Guogang Sun, Jianyi Chen (2016). 'Experimental and CFD study of particle deposition on the outer surface of vortex finder of a cyclone separator' - Chemical Engineering Journal PII: S1385-8947(16)314206.

[50]Le Van Sy (2016). 'Influence of Inlet Angle on Flow Pattern and Performance of Gas-liquid Cylindrical Cyclone Separator' - Particulate Science and Technology ISSN: 0272-6351.

[51]P.A. Funk, K. Elsayed, K.M. Yeater, G.A. Holt, D.P.Whitelock (2015). 'Could cyclone performance 


\section{International Journal of Engineering Applied Sciences and Technology, 2020 \\ Vol. 4, Issue 11, ISSN No. 2455-2143, Pages 312-328 \\ Published Online March 2020 in IJEAST (http://www.ijeast.com)}

improve with reduced inlet velocity?' - Powder Technology280 (2015) 211-218.

[52]F. Justin Dhiraviam, V.Naveenprabhu, M.Santhosh," Study the Effects of Solar Assisted Vapour Compression Air Conditioning System for Winter Applications", International Journal for Scientific Research \& Development, Vol 4(11), (2017), pp. 505-508

[53] V. NaveenPrabhu, K. SaravanaKumar, T. Suresh and M. Suresh," Experimental investigation on tube-intube heat exchanger using nanofluids", Advances in Natural and Applied Sciences, Vol 10(7),(2016), pp. 272-278

[54] V Naveenprabhu, D Mugeshkumaar, KB Pravin, V Ranjith, S Sanjay Arthanari Swamy," A Review of Evaporative Cooling of Finned and Non-Finned Heat Exchanger on Condenser", International Journal for Scientific Research \& Development, Vol 6(2),(2018), pp. 459-461.

[55] V. Naveenprabhu, F.JustinDhiraviam, A.Vimal, K.Kumarrathinam," Design Of Common Header Line For Reduction Of Process Time In Pump Testing”, International Research Journal of Engineering and Technology, Vol 4(1),(2017), pp. 969-975.

[56] B.Santhosh Kumar, et.al," Effect of Load on Joint Efficiency and Hardness in Friction Stir Welding of AA6061 \& AA6063 Aluminium Alloys.", International Journal for Scientific Research \& Development|, Vol 6(2),(2018), pp. 2669-2771.

[57] S. Ganesh Kumar\&V. Thirunavukkarasu (2016). 'Investigation of Tool Wear and Optimization of Process Parameters in Turning of EN8 and EN 36 Steels Asian Journal of Research in Social Sciences and Humanities'. Vol. 6, no.11, pp. 237 - 243, Impact Factor: 0.315.

[58] Pranas Baltrenas, Mantas Pranskevicius, AlbertasVenslovas (2015). 'Optimization of the new generation multichannel cyclone cleaning Efficiency' - ScienceDirect72 (2015) 188 - 195.

[59] Xiaoming Luo, Yibin Wang, Limin He, GengGeng (2015). 'CFD simulation of slug's dissipation for the inlet pipeline of cylindrical cyclone separator' ScienceDirect126 (2015) 481 - 485.

[60]L.S. Brar, R.P. Sharma (2015). 'Effect of varying diameter on the performance of industrial-scale gas cyclone dust separators' - ScienceDirect2 (2015) $3230-3237$.

[61]Lu Duan, XiaolinWu, ZhongliJi, QixianFang (2015). 'Entropy generation analysis on cyclone separators with different exit pipe diameters and inlet dimensions' - Chemical Engineering Science138(2015)622-633.

[62] Sakura GanegamaBogodage, Andrew Y.T. Leung (2015). 'CFD simulation of cyclone separators to reduce air pollution' - Powder Technology286 (2015) 488-506.

[63] Jeongseog Oh, Sangil Choi, Jeonggeun Kim (2015).' Numerical simulation of an internal flow field in a uniflow cyclone separator' - Powder Technology274 (2015) 135-145.

[64] S. D. Kumar, S. S. Kumar\&K. A. Kumar (2018). Investigation of Forced Frequency in a Commercial Vehicle Suspension System. Mechanics and Mechanical Engineering, 22(4), 967-974.

[65] S. Balasubramani\&N. Balaji (2016). Investigations of vision inspection method for surface defects in image processing techniques-a review. Advances in Natural and Applied Sciences, 10(6 SE), 115-120.

[66] S. Balasubramani, K. P. Dhanabalakrishnan, N. Balaji(2015) Optimization of Machining parameters in Aluminium HMMC using Response Surface Methodology. International journal of applied engineering research, 10(20), 19736-19739.

[67] B. Subramaniam, B. Natarajan, B.Kaliyaperumal\&S. J. S. Chelladurai(2018). Investigation on mechanical properties of aluminium 7075-boron carbide-coconut shell fly ash reinforced hybrid metal matrix composites. China Foundry, 15(6), 449-456.

[68] Y. Sureshbabu\&P. AshokaVarthanan. Study the emission characteristics of catalytic coated piston and combustion chamber of a four-stroke spark ignition (SI) engine. Journal of Chemical and Pharmaceutical Sciences ISSN, 974, 2115.

[69] Y. Sureshbabu\&P. AshokaVarthanan (2018) Study the emission characteristics of a catalytic coated piston and combustion chamber of a four-stroke spark ignition (SI) engine. International Journal for Scientific Research \& Development, 6(02), 19811983.

[70]Z. Qi, S.B. Kuang, A.B. Yua (2015). 'Numerical investigation of the separation behaviors of fine particles in large dense medium cyclones' International Journal of Mineral Processing $\mathrm{xxx}$ (2015) xxx-Xxx.

[71]Rainier Hreiz, Richard Lainé, Jing Wu, Cécile Lemaitre, Caroline Gentric, Denis Fünfschilling (2014). 'On the effect of the nozzle design on the 
performances of gas-liquid cylindrical cyclone separators' - International Journal of Multiphase Flow58 (2014) 15-26.

[72] VladasVekteris, VytautasStrishka, Darius Ozarovskis, Vadim Mokshin(2014).' Experimental investigation of processes in acoustic cyclone separator 'Advanced Powder Technology25 (2014) $1118-1123$.

[73] Sujeet Kumar Shukla, Prashant Shukla, Pradyumna Ghosh (2013). 'The effect of modeling of velocity fluctuations on the prediction of collection efficiency of cyclone separators' - Applied Mathematical Modelling37 (2013) 5774-5789.

[74]Lian-ping Dong, Min-qiang Fan, Hong-li Yang (2013). 'Separation performance of a cyclone column separator with complicated positive and negative cones' - International Journal of Mineral Processing122 (2013) 43-46.

[75]Jingxuan Yang, Guogang Sun, Cuizhi Gao (2013). 'Effect of the inlet dimensions on the maximumefficiency cyclone height' - Separation and Purification Technology105 (2013) 15-23. 